\title{
Fatty Acid $\beta$-Oxidation-dependent and -independent Responses and Tumor Aggressiveness Acquired Under Mild Hypoxia
}

\author{
MASATOSHI WAKUI ${ }^{1}$, KENJI KAWAI ${ }^{2}$, TOMOKO MIZUSHIMA ${ }^{2}$, CHIYOKO NISHIME $^{2}$, \\ AKIHIKO SERIZAWA ${ }^{3}$, HIROSHI SUEMIZU ${ }^{2}$, KEISUKE ASAKURA ${ }^{4}$, YOSHIKANE YAMAUCHI $^{5}$, \\ TETSU HAYASHIDA ${ }^{6}$, MAKOTO SUEMATSU ${ }^{7}$ and MITSURU MURATA ${ }^{1}$ \\ Departments of ${ }^{1}$ Laboratory Medicine, ${ }^{6}$ Surgery, and ${ }^{7}$ Biochemistry, and \\ ${ }^{4}$ Division of Thoracic Surgery, Keio University School of Medicine, Tokyo, Japan; \\ ${ }^{2}$ Central Institute for Experimental Animals, Kawasaki, Japan; \\ ${ }^{3}$ Division of Diagnostic Pathology, Tokai University Hospital, Isehara, Japan; \\ ${ }^{5}$ Department of Surgery, Teikyo University School of Medicine, Tokyo, Japan
}

\begin{abstract}
Background/Aim: The present study assessed whether and how tumor cells undergoing hypoxia contribute to disease progression after moving to areas with different oxygen conditions. Materials and Methods: Human colorectal carcinoma HCT116 cells cultured under mild hypoxia were subjected to in vivo experiments using transfer to immunodeficient murine recipients and to in vitro experiments using pharmacological inhibition of fatty acid $\beta$-oxidation (FAO). Results: Bone involvement and hepatic metastases were accelerated in transfer models of hypoxically cultured HCT116 cells. Hypoxic HCT116 cells exhibited FAO-dependent glycogen synthesis. FAO-dependent and-independent induction of gene expression also occurred under hypoxia. The distribution of glucose transporter 1 expression compared with heme oxygenase 1 expression in HCT116 cell spheroids seemed consistent with differential dependence of hypoxic expression of these molecules on FAO. Conclusion: These results provide insights into the contribution of hypoxia to tumor progression and the relevance of FAO.
\end{abstract}

A number of reports have mentioned biological or clinical relevance of hypoxia to tumor progression results in treatment-refractory courses and poor prognoses. However, the mechanisms of how hypoxia gives rise to tumor progression and resistance to therapies remain to be

Correspondence to: Masatoshi Wakui, Department of Laboratory Medicine, Keio University School of Medicine, 35 Shinanomachi, Shinjuku-ku, Tokyo 160-8582, Japan. E-mail: wakuism@a6.keio.jp

Key Words: Hypoxia, fatty acid $\beta$-oxidation, human colorectal carcinoma HCT116 cells, tumor aggressiveness, glycogen synthesis, transcriptomic profiles, cell spheroids. elucidated $(1,2)$. For example, it is still unclear whether once surviving hypoxia tumor cells play a role in tumor progression even after moving to areas with different oxygen conditions. It is of considerable interest to clarify the effects of exposure to mild hypoxia, where tumor cells can survive, on tumor growth, invasion, and metastases.

To assess whether and how tumor cells once exposed to hypoxia subsequently contribute to disease progression, human colorectal carcinoma HCT116 cells cultured under mild hypoxia were transferred to immunodeficient mice and subjected to macroscopic and microscopic observations.

\section{Materials and Methods}

Cell culture. HCT116 human colorectal carcinoma cell line was obtained from the American Type Culture Collection (Manassas, VA, USA). HCT116 cells were maintained in McCoy's 5A (SigmaAldrich, St. Louis, MO, USA) containing antibiotics and $10 \%$ fetal bovine serum (FBS) (HyClone, Logan, UT, USA). For normoxic culture for 7 days, HCT116 cells were incubated in a humidified $\left(37^{\circ} \mathrm{C}, 5 \% \mathrm{CO}_{2}\right)$ incubator. For hypoxic culture, HCT116 cells were incubated in an AnaeroPack Anaero cultivation system with AnaeroPack MicroAero (Mitsubishi Gas Chemical, Japan), which provides $6-12 \% \mathrm{O}_{2}$ conditions. To pharmacologically inhibit fatty acid $\beta$-oxidation (FAO) during culture, etomoxir (Sigma-Aldrich) was added into medium at the concentration of $60 \mathrm{mM}$.

Ethics statement for animal experimentation. The in vivo experiments were performed in accordance with the institutional guideline and approved by the Animal Experimentation Committee of the Keio University and the Central Institute for Experimental Animals (approval number 062070).

Subcutaneous tumor formation. To form subcutaneous tumors, $1 \times 10^{4}$ HCT116 cells, which had been cultured normoxically or hypoxically, in $0.2 \mathrm{ml}$ of serum-free medium were transferred to NOD/SCID/IL2R $\gamma c^{\text {null }}$ (NOG) mice subcutaneously. Palpable tumors were measured half-weekly, and the recipients were 
sacrificed for anatomical and histological examination 6 weeks after the transfer.

Liver metastasis induction. Liver metastases were induced by intrasplenic transfer of $1 \times 10^{4} \mathrm{HCT} 116$ cells to each of $11 \mathrm{NOG}$ mice (six normoxic cell transfer recipients and five hypoxic cell transfer recipients), followed by splenectomy as described previously (3). The recipients were sacrificed for macroscopic evaluation of liver metastases 6 weeks after the transfer.

Immunostaining of sections from subcutaneous tumors. Sections (3- $\mu \mathrm{m}$-thick) were cut from paraffin-embedded tissue blocks. Morphology of tissue sections was determined with hematoxylin-eosin (H\&E) staining. Immunostaining of the sections with a mouse monoclonal antibody to human E-cadherin (clone NCH-38; Dako, Glostrup, Denmark), a mouse monoclonal antibody to human vimentin (clone V9; Dako), or a rabbit polyclonal antibody to human S100 calcium-binding protein A4 (S100A4) (Dako) was performed on the Bond-Max automated IHC platform (Leica Biosystems, Mount Waverley, Australia). Photographic images of the immunostained sections were prepared by Axio Imager M1 (Carl Zeiss, Thornwood, NY, USA) and subjected to comparison of visualized expression distribution of E-cadherin, vimentin, and S100A.

Transmission electron microscopy. HCT116 cells cultured under normoxia or hypoxia were rinsed with phosphate-buffered saline. Cells were initially fixed with $2.5 \%$ glutaraldehyde in $0.1 \mathrm{M}$ sodium phosphate buffer ( $\mathrm{pH} 7.4)$, and then post-fixed in $0.1 \mathrm{M}$ sodium phosphate buffer containing $1 \%$ osmium tetroxide $(\mathrm{pH} \mathrm{7.2)}$ for $2 \mathrm{~h}$ at $4^{\circ} \mathrm{C}$. After being dehydrated in a graded series of ethanol, substituted by acetone, cells were embedded into Quetol 812 epoxy resin (NissinEMCo., Tokyo, Japan). Ultrathin ( $90 \mathrm{~nm})$ sections were cut on an Ultratome III (LKB, Bromma, Sweden) and double stained with $1 \%$ uranyl acetate sol and lead citrate sol. Images were obtained by electron microscopy (JEM-1200EX; JEOL, Tokyo, Japan) and subjected to comparison of morphological features between normoxic and hypoxic HCT116 cells at $\times 2,000$ and $\times 5,000$ magnification.

Periodic acid-Schiff (PAS) staining. To cytochemically assess whether glycogen was accumulated in HCT116 cells cultured hypoxically but not normoxically, PAS staining was performed as previously reported (4) and subjected to morphological observations of normoxic and hypoxic HCT116 cells.

Glycogen measurement assay. For analysis of the amount of glycogen in HCT116 cells cultured normoxically or hypoxically with or without FAO inhibition by etomoxir, enzymatic hydrolysis with amylo- $\alpha-1,4-\alpha-1,6$-glucosidase followed by measurement of the resultant glucose was carried out as previously described (5).

Transcriptomic analysis. Total RNAs were extracted from collected HCT116 cells using the TRIzol reagent (Invitrogen, Carlsbad, CA, USA) and subjected to synthesis of complementary RNAs (cRNAs). Cyanine 3-labeled cRNAs were synthesized using QuickAmp Labeling Kits (Agilent Technologies). The cRNAs were hybridized at $65^{\circ} \mathrm{C}$ for $17 \mathrm{~h}$ to Whole Human Genome $44 \mathrm{~K}$ Microarrays (Agilent Technologies). After being washed, the microarrays were scanned using an Agilent DNA microarray scanner (Agilent Technologies). Intensity values of each scanned feature were quantified using Agilent Feature Extraction software (Agilent Technologies). The raw microarray data are deposited in the National Center for Biotechnology Information Gene Expression Omnibus database under accession number GSE109318. Rank-based quantile normalization was applied to the raw signal data using $\mathrm{R}$ software version 3.0.2. Probes were filtered based on pre-existing annotation with gene symbol and signal intensity. For genes with more than one probe, the probe that had the highest signal intensity was adopted.

Cell spheroid formation. The spheroid culture for 1-4 weeks was carried out using the Sumilon Celltight Spheroid 96U (Sumitomo Bakelite, Tokyo, Japan) with McCoy's 5A medium containing antibiotics and $10 \% \mathrm{FBS}$ in a humidified $\left(37^{\circ} \mathrm{C}, 5 \% \mathrm{CO}_{2}\right)$ incubator.

Immunostaining of sections from cell spheroids. Sections (3- $\mu$ mthick) were cut from paraffin-embedded cell spheroid blocks. Morphology of cell spheroid sections was determined with H\&E staining. Immunostaining of the sections with mouse monoclonal antibody to human Ki-67 antigen (clone MIB-1; Dako), rabbit polyclonal antibody to human heme oxygenase 1 (HO1) (Stressgen Biotechnologies, San Diego, CA, USA), or rabbit polyclonal antibody to human glucose transporter 1 (GLUT1) (Dako) was performed and followed by preparation of photographic images of the immunostained sections for comparison of visualized expression distribution of Ki-67, GLUT1, and HO1, as described above.

Statistical analysis. Two sample $t$-test with Welch's correction, oneway analysis of variance, two-way analysis of variance, MannWhitney test, or Fisher's exact probability test was used to statistically analyze the data. $p$-Values less than 0.05 were considered significant.

\section{Results}

Hypoxic culture makes HCT116 cells acquire tumor aggressiveness. Tumor sizes tended to be larger after the subcutaneous transfer of hypoxically cultured HCT116 cells than after that of normoxically cultured HCT116 cells (Figure 1A), although without statistical significance. Most strikingly, rib involvement due to tumor invasion was observed in all the recipients of hypoxically cultured HCT116 cells but in none of the recipients of normoxically cultured HCT116 cells $(p<0.003$, Figure 1B). Liver metastases were increased after the intrasplenic transfer of hypoxically cultured HCT116 cells, compared with those after the intrasplenic transfer of normoxically cultured HCT116 cells ( $p<0.01$, Figure 2).

As shown in Figure 3, the distribution of E-cadherin and that of vimentin expression appeared to be contrasting in both the subcutaneous tumors formed by normoxically cultured HCT116 cells and in those formed by hypoxically cultured HCT116 cells, while their distribution appeared to be less dense in the tumors of hypoxically cultured HCT116 cells. The expression distribution of S100A4 was more obvious in the tumors of hypoxically cultured HCT116 cells than in the tumors of normoxically cultured ones and overlapped partly with that of E-cadherin and vimentin in the tumors of hypoxically but not normoxically cultured HCT116 cells. 
A

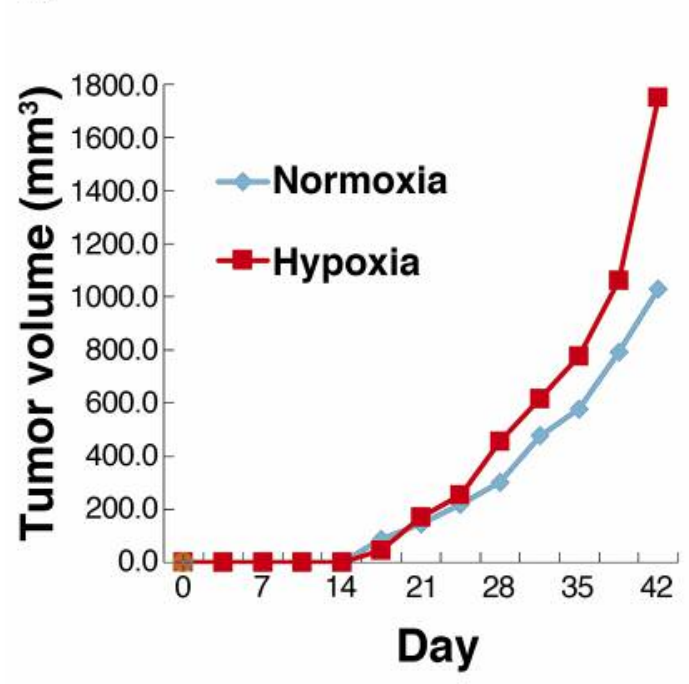

B

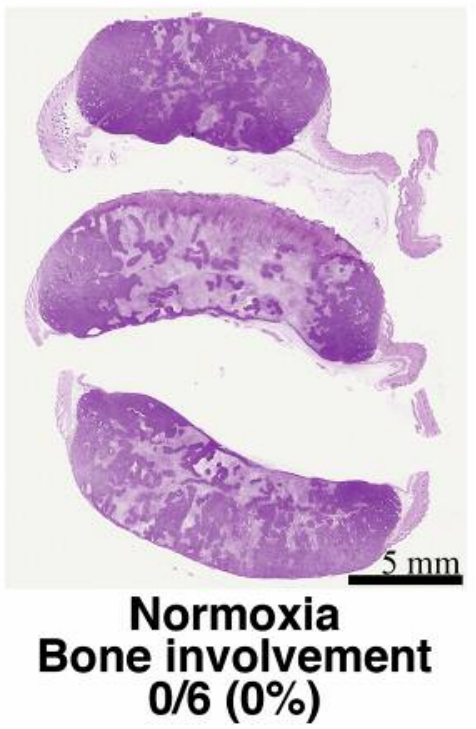

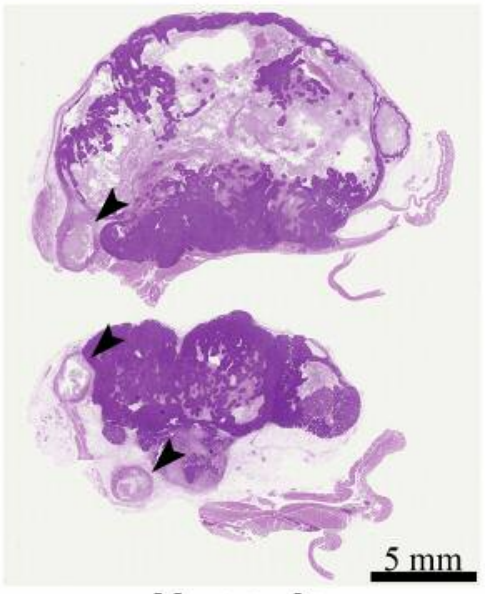

Hypoxia

Bone involvement $6 / 6(100 \%)$

Figure 1. Comparison of findings in recipients after subcutaneous transfer of normoxically ( $n=6)$ versus hypoxically ( $n=6)$ cultured HCT116 cells. A: Comparison of mean tumor volume between the two recipient groups is shown. The tumor sizes tended to be larger after the transfer of hypoxically cultured HCT116 cells, although the differences were not statistically significant during the observation period. B: Rib involvement occurred in all the recipients after the transfer of hypoxically cultured HCT116 cells but in none of those after the transfer of normoxically cultured HCT116 cells ( $p<0.003$ ). The bone involvement was histologically confirmed. The representative results of hematoxylin-eosin staining are shown. Arrowheads indicate bone involvement.
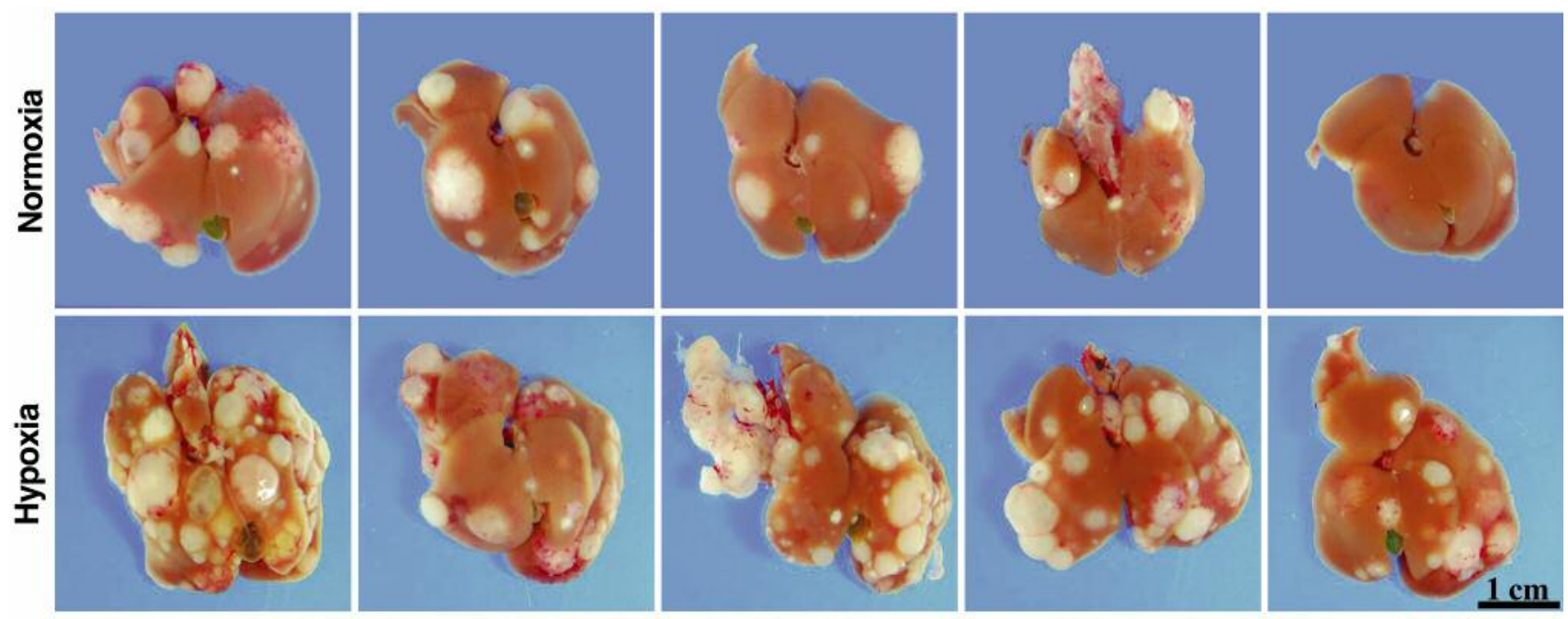

Figure 2. Comparison of liver metastases in recipients after intrasplenic transfer of normoxically ( $n=6)$ versus hypoxically $(n=5)$ cultured HCT116 cells. Liver metastases were macroscopically observed in five of six recipients of normoxically cultured HCT116 cells and in all five those which received hypoxically cultured HCT116 cells. Findings of livers from the 10 recipients exhibiting macroscopic metastases are shown. The numbers of metastatic foci were increased after the intrasplenic transfer of hypoxically cultured HCT116 cells $(p<0.01)$.

Hypoxic culture makes HCT116 cells increase glycogen synthesis dependently on FAO. As shown in Figure 4A, the electron microscopy demonstrated a considerable amount of glycogen, suggesting that HCT116 cells increase glycogen synthesis in response to hypoxia as muscle cells do, dependently on FAO but not on glycolysis. The positivity for PAS staining also supported this observation (Figure 4B). As expected, the increase of glycogen synthesis in hypoxically 

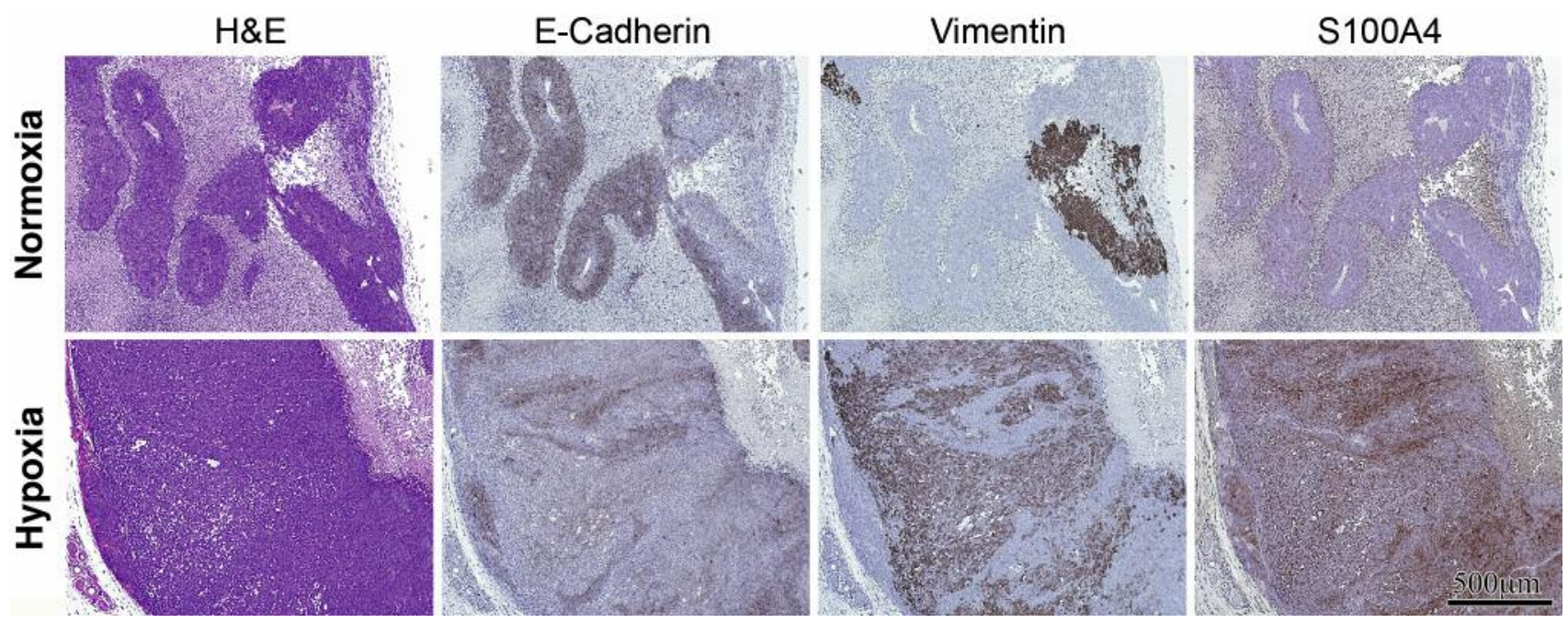

Figure 3. Comparison of histological findings of subcutaneous tumors formed after normoxically versus hypoxically cultured HCT116 cells. Representative results of hematoxylin-eosin $(H \& E)$ staining and immunostaining are shown.

A
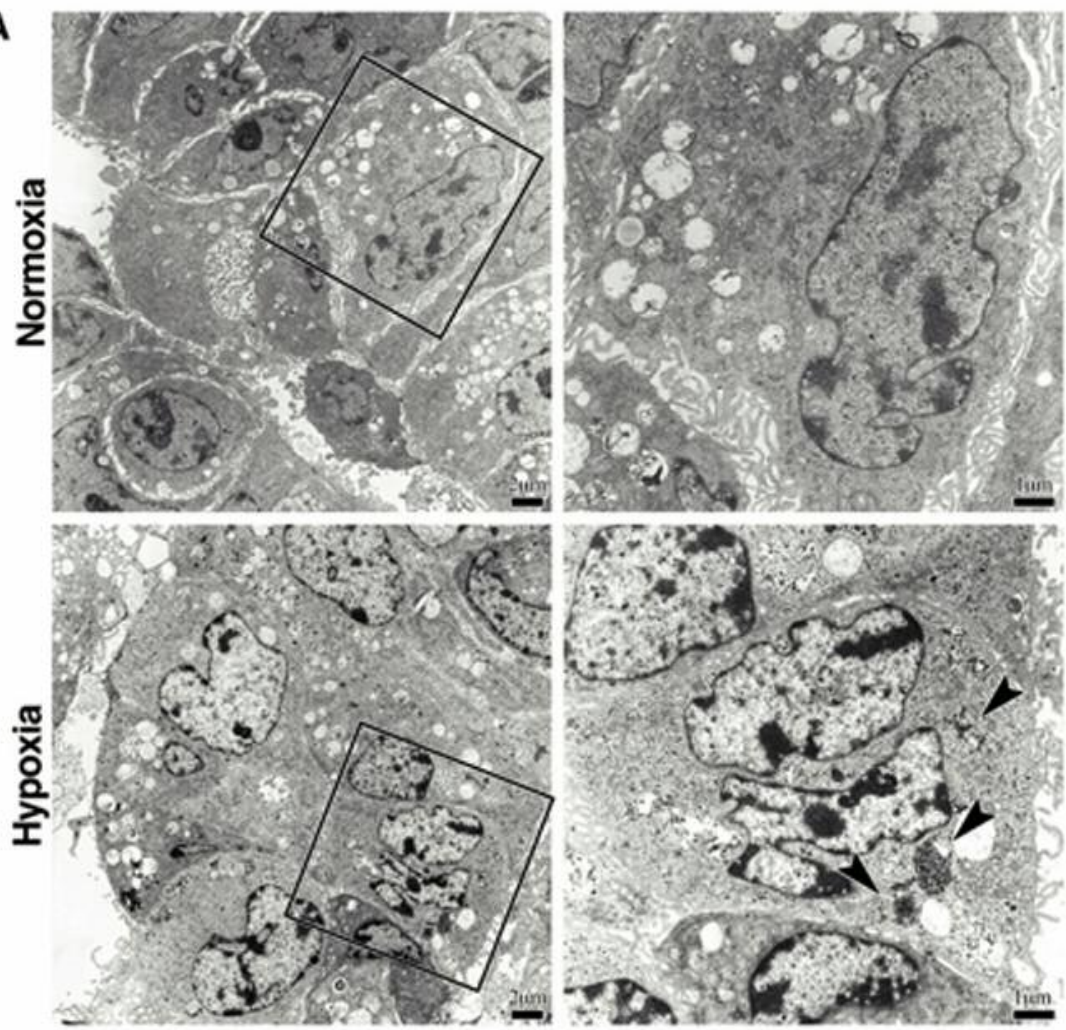

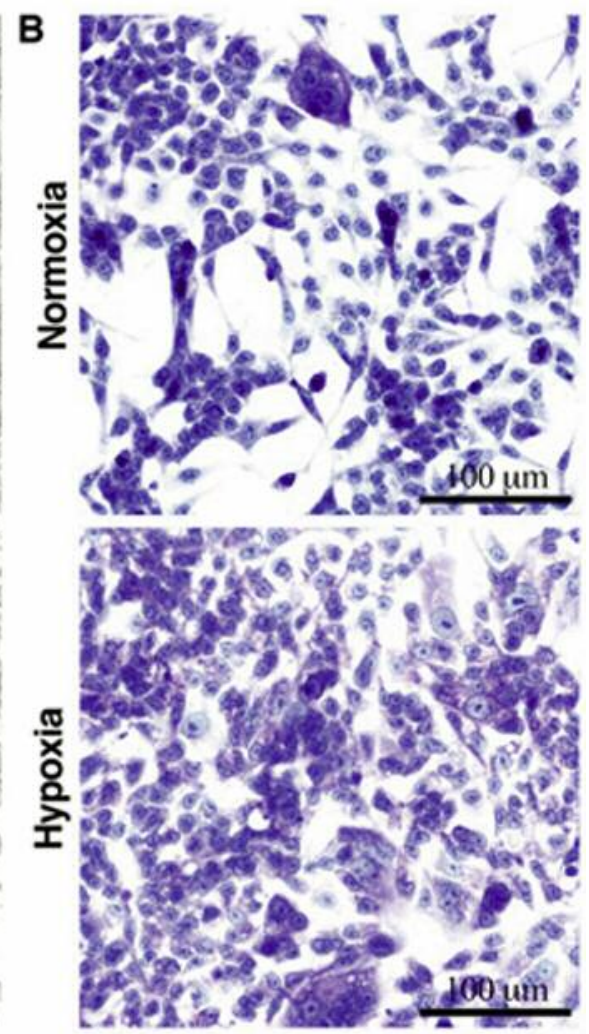

Figure 4. Comparison of electron microscopic and cytochemical findings of normoxically versus hypoxically cultured HCT116 cells. A: Glycogen synthesis appeared to be increased by hypoxia according to electron microscopic observations. Left: Images show representative electron micrographs of normoxically and hypoxically cultured HCT116 cells, respectively, at ×2,000 magnification. Right: Images of inset areas at higher magnification $(\times 5,000)$. Arrowheads indicate glycogen accumulation. B: Periodic acid-Schiff staining also supported suggestion by electron microscopic findings. Representative results are shown. 

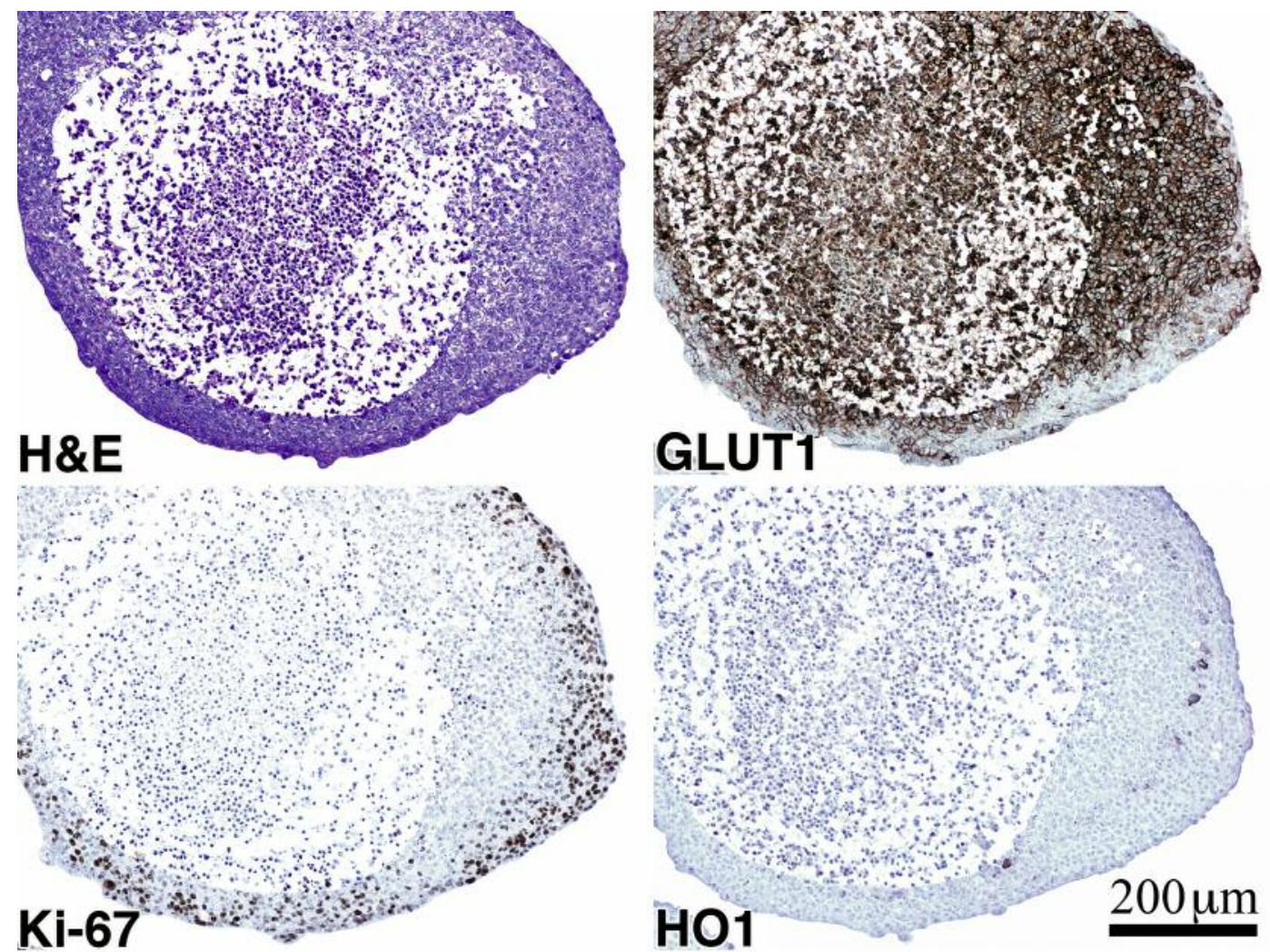

Figure 5. Immunostaining of sections from HCT116 cell spheroids. The distribution of glucose transporter 1 (GLUT1) expression in the HCT116 cell spheroids was suggestive of hypoxic areas, in contrast to that of Ki-67 expression. A limited number of cells expressing heme oxygenase 1 (HO1) were located at the margin of areas staining positively for GLUT1 expression. Representative results of hematoxylin-eosin (H\&E) staining and immunostaining are shown.

cultured HCT116 cells was prevented by pharmacological inhibition of FAO using etomoxir, suggesting a role for FAO in hypoxic adaptation $(p<0.01$, Table I).

Transcriptomic profiles reveal FAO-dependent and -independent gene expression induced by hypoxia. As indicated in Tables II and III, transcriptomic characterization of HCT116 cells cultured hypoxically or normoxically with or without pharmacological inhibition of FAO revealed that FAO-dependent and independent gene expression occurred in response to hypoxia. The former included not only simple induction via FAOdependent mechanisms but also complicated induction both by hypoxia via FAO-dependent mechanisms and by FAO impairment via oxygen-dependent mechanisms (Table II). It is notable that there were no genes exhibiting expression induced by FAO impairment via oxygen-independent mechanisms.

Spheroids exhibit hypoxic expression of molecules differentially depending on FAO. In vitro spheroids exhibit internal heterogeneity regarding oxygen concentration, and more closely represent in vivo tumours than cells cultured
Table I. Glycogen amounts in cultured HCT116 cells.

\begin{tabular}{lcc}
\hline Oxygen & Etomoxir & Mean glycogen $\left(\mathrm{mg} / \times 10^{6}\right.$ cells $)$ \\
\hline Normoxia & - & 0.217 \\
& + & 0.221 \\
Hypoxia & - & $3.03^{*}$ \\
& + & 0.247 \\
\hline
\end{tabular}

*Significantly different at $p<0.01$ ( $\mathrm{n}=3$ for each group).

under different external oxygen conditions (6-8). To ascertain the association of dependence of hypoxic expression of molecules on FAO with graded hypoxia, immunostaining of GLUT1 and HO1 was performed in HCT116 cell spheroids (Figure 5). It is well known that expression of solute carrier family 2 (facilitated glucose transporter), member 1 (SLC2A1) gene encoding GLUT1 is induced by hypoxia via hypoxia-inducible factor $1 \alpha$ (HIF1 $\alpha$ ) signaling regardless of FAO activity, as supported by the transcriptomic data (Table 
III). On the other hand, HMOX1 gene encoding HO1 is one of many genes exhibiting expression induced by hypoxia via FAO-dependent mechanisms as revealed by the transcriptomic data (Table II). As expected, the distribution of GLUT1 expression in the HCT116 spheroids was suggestive of hypoxic areas, in contrast to that of Ki-67 expression. Of particular interest, a limited number of cells expressing HO1 were located at the margin of areas staining positive for GLUT1 expression.

\section{Discussion}

The present study provided several lines of evidence for the acquirement of tumor aggressiveness due to hypoxia and for relevance of FAO to hypoxic adaptation of HCT116 cells. Occurrence of bone involvement and a tendency for promotion of tumor growth after subcutaneous transfer as well as increase of liver metastases after intrasplenic transfer demonstrated that the aggressive features acquired by tumor cells undergoing hypoxia are preserved even after the oxygen level is restored.

To assess whether acquisition of tumor aggressiveness was made via epithelial-mesenchymal transition (EMT), the expression profiles of three EMT-associated molecules were evaluated by immunostaining of sections from subcutaneous tumors. As E-cadherin and vimentin are well known as negative and positive EMT markers, respectively, the contrasting distribution of these molecules was consistent (Figure 3) (9). On the other hand, the distribution of expression appeared to be less dense in the tumors of hypoxically cultured HCT116 cells. It still remains to be elucidated how such differences in the distribution density of EMT markers is relevant to differences in tumor progression. S100A4 has been reported to induce EMT, maintain stemness, and enhance liver metastases $(10,11)$. The higher expression of S100A4 in tumors formed after the subcutaneous transfer of hypoxically cultured HCT116 cells and the increased foci of liver metastases occurring after the intrasplenic transfer of those seems consistent with a previous report on enhancement of liver metastases by S100A4 overexpression (10). To clarify the role for EMT in tumor aggressiveness acquired under mild hypoxia, more findings should be accumulated.

The absence of gene expression induced by FAO inhibition via oxygen-independent mechanisms is worthy of note. Reduced flavin adenine dinucleotide $\left(\mathrm{FADH}_{2}\right)$ and reduced nicotinamide adenine dinucleotide (NADH) produced by FAO or by tricarboxylic acid (TCA) cycles consuming acetyl-CoA from FAO, supply electrons for ATP production by oxidative phosphorylation in mitochondria. Under impaired FAO, the TCA cycle consuming metabolites from catabolism of glucose or amino acids would be promoted by gene expression via oxygen-dependent mechanisms. In the present study, SLC25A42 gene expression was induced both by hypoxia via FAO dependent mechanisms and by FAO impairment via oxygen dependent mechanisms (Table II). Of interest, this gene encodes a mitochondrial transporter of CoA to support a number of fundamental processes of the metabolism in mitochondria (12). Such induction of $S L C 25 A 42$ gene expression seems to compensate for hypoxia dependently on FAO or that for impaired FAO dependently on enough oxygen to maintain mitochondrial activity. In contrast, combination of FAO inhibition and hypoxia would prevent ATP production by oxidative phosphorylation and lead to energy generation based exclusively on glycolysis independently of mitochondria. Thus, under mild hypoxia, in which FAO can still function, gene transcripts might be induced via FAOdependent mechanisms for adaptive responses.

Glycogen synthesis occurring dependently on FAO under hypoxia seems reasonable. It is plausible that stored glycogen is consumed as the source of substrate of glycolysis for energy generation under hypoxia becoming severe enough to inactivate mitochondrial energy metabolism or under mild hypoxia accompanied by FAO impairment. Of note, glycogen accumulation under hypoxia has been reported to be associated with increased expression of enzymes promoting glycogen synthesis, such as glycogen synthase 1 (GYS1) and protein phosphatase 1 regulatory subunit 3C (PPP1R3C) (4, 13). Similarly, expression of PPP1R3G, which promotes glycogen synthesis like PPP1R3C, was induced by hypoxia via FAO-independent mechanisms in the present study (Table III) (14). However, the reduction of hypoxic glycogen synthesis by pharmacological inhibition of FAO suggests that glycogen accumulation occurring in hypoxic HCT116 cells would result from the combination of activation of glycogen synthase and inactivation of glycogen phosphorylase via allosteric effects and protein dephosphorylation in the presence of sufficient ATP supplied by FAO (15). In contrast to mild hypoxia, severe hypoxia would allow HCT116 cells to utilize only mitochondria-independent metabolism for energy generation, resulting in limitation of cell survival. In fact, HCT116 cells cannot survive for more than 2 or 3 days under less than $1 \%$ oxygen (data not shown).

In the HCT116 cell spheroids, a limited number of cells expressing $\mathrm{HO} 1$ encoded by the HMOXI gene were located at the margin of areas that were rich in cells expressing GLUT1 encoded by $S L C 2 A 1$ gene. Hypoxic expression of SLC2Al gene does not depend on FAO, while hypoxic expression of HMOX1 gene depends on FAO. It is plausible that hypoxia at such locations appeared to be mild. HO1 expression may represent adaptive responses to hypoxia still allowing tumor cells to maintain FAO. It is likely that hypoxia which becomes so severe as to prevent FAO results in loss of HO1 expression. Thus, HO1 expression might be 
Table II. Genes exhibiting expression by simple induction via fatty acid $\beta$-oxidation (FAO)-dependent mechanisms, and complex induction both by hypoxia via FAO-dependent mechanisms and by FAO impairment via oxygen-dependent mechanisms.

\begin{tabular}{|c|c|c|}
\hline Induction & Gene symbol & Gene name \\
\hline \multirow[t]{13}{*}{ Simple } & $A T P 2 B 4$ & ATPase, $\mathrm{Ca}^{++}$transporting, plasma membrane 4 \\
\hline & RHCE & $\mathrm{Rh}$ blood group, $\mathrm{CcEe}$ antigens \\
\hline & $P S C A$ & Prostate stem cell antigen \\
\hline & SNTA1 & Syntrophin, alpha 1 (dystrophin-associated protein $\mathrm{A} 1,59 \mathrm{kDa}$, acidic component) \\
\hline & FAM46B & Family with sequence similarity 46 , member B \\
\hline & DGCR5 & DiGeorge syndrome critical region gene 5 (non-protein coding) \\
\hline & DKFZp451A211 & DKFZp451A211 protein \\
\hline & GSTA2 & Glutathione S-transferase alpha 2 \\
\hline & CTRC & Chymotrypsin C (caldecrin) \\
\hline & DUSP7 & Dual specificity phosphatase 7 \\
\hline & SOCS1 & Suppressor of cytokine signaling 1 \\
\hline & $F A M 222 B$ & Family with sequence similarity 222 member B \\
\hline & SNX21 & Sorting nexin family member 21 \\
\hline \multirow[t]{26}{*}{ Complex } & $S L C 25 A 42$ & Solute carrier family 25, member 42 \\
\hline & $P D K 4$ & Pyruvate dehydrogenase kinase, isozyme 4 \\
\hline & GCOM1 & GRINL1A complex locus \\
\hline & CD52 & CD52 molecule \\
\hline & $H A S 3$ & Hyaluronan synthase 3 \\
\hline & DUSP13 & Dual specificity phosphatase 13 \\
\hline & $S D C B P 2$ & Syndecan binding protein (syntenin) 2 \\
\hline & HMOX1 & Heme oxygenase (decycling) 1 \\
\hline & KRTAP3-1 & Keratin associated protein 3-1 \\
\hline & EBI3 & Epstein-Barr virus induced 3 \\
\hline & $D A P P 1$ & Dual adaptor of phosphotyrosine and 3-phosphoinositides \\
\hline & $A B C C 2$ & ATP-binding cassette, sub-family C (CFTR/MRP), member 2 \\
\hline & $L Y 6 K$ & Lymphocyte antigen 6 complex, locus $\mathrm{K}$ \\
\hline & RHOF & Ras homolog gene family, member F (in filopodia) \\
\hline & TMEM139 & Transmembrane protein 139 \\
\hline & $A C C S L$ & 1-Aminocyclopropane-1-carboxylate synthase homolog (Arabidopsis)(non-functional)-like \\
\hline & $F T L$ & Ferritin, light polypeptide \\
\hline & KRT7 & Keratin 7 \\
\hline & SECTM1 & Secreted and transmembrane 1 \\
\hline & PLIN2 & Perilipin 2 \\
\hline & ACOT11 & Acyl-CoA thioesterase 11 \\
\hline & TRIM16L & Aripartite motif-containing 16-like \\
\hline & $A K R 1 C 3$ & Aldo-keto reductase family 1 , member $\mathrm{C} 3$ (3-alpha hydroxysteroid dehydrogenase, type II) \\
\hline & LOC100133263 & Hypothetical LOC100133263 \\
\hline & GRHL3 & Grainyhead-like 3 (Drosophila) \\
\hline & GRAMD3 & GRAM domain containing 3 \\
\hline
\end{tabular}

utilized as a biomarker for grading of hypoxia with respect to adaptive responses by tumor cells. Taken together, the distribution of cells expressing GLUT1 compared with cells expressing HO1 in HCT116 cell spheroids might reflect the presence of graded hypoxia associated with differential dependence on FAO.

The relevance of FAO to hypoxic adaptation is of considerable interest. According to the transcriptomic profiles, potential compensation of failure of FAO by other mitochondrial pathways requires normoxic conditions. On the other hand, there were gene transcripts induced by hypoxia via FAO-dependent mechanisms, such as SLC2Al.
Thus, compared with other mitochondrial pathways, FAO seems more resistant to hypoxia and results in energy generation to enable glycogen synthesis. This may reflect a critical role for FAO in tumor adaptation to hypoxia. A report on targeting hypoxic cancer stem cells with doxycycline presented evidence that mitochondrial biogenesis metabolically linking to FAO is required for their propagation induced by hypoxia (16), supporting the idea regarding the significance of FAO suggested by the present study. More recently, Iwamoto et al. demonstrated that tumor cells switch from glycolysis to FAO upon treatment with antiangiogenic drug, providing evidence for the 
Table III. Genes exhibiting hypoxic and fatty acid $\beta$-oxidation (FAO)-independent expression.

\begin{tabular}{|c|c|}
\hline Gene symbol & Gene name \\
\hline$P D K 1$ & Pyruvate dehydrogenase kinase, isozyme 1 \\
\hline C8orf58 & Chromosome 8 open reading frame 58 \\
\hline C4orf3 & Chromosome 4 open reading frame 3 \\
\hline$A D M$ & Adrenomedullin \\
\hline EROIL & ERO1-like (Saccharomyces cerevisiae) \\
\hline LOXL2 & Lysyl oxidase-like 2 \\
\hline LOC390595 & Similar to ubiquitin-associated protein 1 (predicted) \\
\hline$P D G F A$ & Platelet-derived growth factor alpha polypeptide \\
\hline ILIRAP & Interleukin 1 receptor accessory protein \\
\hline LOC100132805 & Hypothetical LOC100132805 \\
\hline$I K B K B$ & Inhibitor of kappa light polypeptide gene enhancer in B-cells, kinase beta \\
\hline SPRYI & Sprouty homolog 1, antagonist of fibroblast growth factor signaling (Drosophila) \\
\hline ZNF292 & Zinc finger protein 292 \\
\hline COL7A1 & Collagen, type VII, alpha 1 \\
\hline CXCR4 & Chemokine (C-X-C motif) receptor 4 \\
\hline GPI & Glucose phosphate isomerase \\
\hline$T N N T 2$ & Troponin T type 2 (cardiac) \\
\hline ARHGAP29 & Rho GTPase activating protein 29 \\
\hline SLCO4A1 & Solute carrier organic anion transporter family, member 4A1 \\
\hline LOC286052 & Hypothetical protein LOC286052 \\
\hline$P M F B P 1$ & Polyamine modulated factor 1 binding protein 1 \\
\hline BHLHE4O & Basic helix-loop-helix family, member e 40 \\
\hline$S L C 2 A 1$ & Solute carrier family 2 (facilitated glucose transporter), member 1 \\
\hline ENO2 & Enolase 2 (gamma, neuronal) \\
\hline CATSPERB & Cation channel, sperm-associated, beta \\
\hline FLJ44342 & Hypothetical LOC645460 \\
\hline RNF207 & Ring finger protein 207 \\
\hline RNF112 & Ring finger protein 112 \\
\hline$S L C 2 A 3$ & Solute carrier family 2 (facilitated glucose transporter), member 3 \\
\hline PCSK9 & Proprotein convertase subtilisin/kexin type 9 \\
\hline GNRH1 & Gonadotropin-releasing hormone 1 (luteinizing-releasing hormone) \\
\hline$H G D$ & Homogentisate 1,2-dioxygenase (homogentisate oxidase) \\
\hline PTGS1 & Prostaglandin-endoperoxide synthase 1 (prostaglandin G/H synthase and cyclooxygenase) \\
\hline EFCAB3 & EF-hand calcium binding domain 3 \\
\hline FAM83E & Family with sequence similarity 83 , member $\mathrm{E}$ \\
\hline$T B C 1 D 3 B$ & TBC1 domain family, member 3B \\
\hline STRC & Stereocilin \\
\hline LOC92973 & Hypothetical LOC92973 \\
\hline NCRNA00173 & Non-protein coding RNA 173 \\
\hline P4HAl & Prolyl 4-hydroxylase, alpha polypeptide I \\
\hline EGLN3 & Egl nine homolog 3 (Caenorhabditis elegans) \\
\hline PRAM1 & PML-RARA regulated adaptor molecule 1 \\
\hline FRMD3 & FERM domain containing 3 \\
\hline$S S C 5 D$ & Scavenger receptor cysteine-rich glycoprotein \\
\hline$B N I P 3 L$ & BCL2/adenovirus E1B $19 \mathrm{kDa}$ interacting protein 3-like \\
\hline$A L D O C$ & Aldolase $\mathrm{C}$, fructose-bisphosphate \\
\hline MEGF6 & Multiple EGF-like-domains 6 \\
\hline CPXM2 & Carboxypeptidase X (M14 family), member 2 \\
\hline NOD2 & Nucleotide-binding oligomerization domain containing 2 \\
\hline CST6 & Cystatin E/M \\
\hline FOSL2 & FOS-like antigen 2 \\
\hline$L D H A$ & Lactate dehydrogenase A \\
\hline$A L D O A$ & Aldolase A, fructose-bisphosphate \\
\hline PFKFB4 & 6-Phosphofructo-2-kinase/fructose-2,6-biphosphatase 4 \\
\hline BNIP3 & BCL2/adenovirus E1B $19 \mathrm{kDa}$ interacting protein 3 \\
\hline C1S & Complement component $1, \mathrm{~s}$ subcomponent \\
\hline$R R P 7 B$ & Ribosomal RNA processing 7 homolog B (S. cerevisiae) \\
\hline CD300A & CD300a molecule \\
\hline
\end{tabular}


Table III. Continued

\begin{tabular}{ll}
\hline Gene symbol & Gene name \\
\hline LOC100128913 & Hypothetical protein LOC100128913 \\
PPP1R3G & Protein phosphatase 1, regulatory (inhibitor) subunit 3G \\
NEAT1 & Nuclear paraspeckle assembly transcript 1 (non-protein coding) \\
TET2 & Tet oncogene family member 2 \\
FUT11 & Fucosyltransferase 11 (alpha $(1,3)$ fucosyltransferase) \\
ARRDC3 & Arrestin domain containing 3 \\
FLJ32224 & Hypothetical gene supported by AK056786 \\
SLC5A3 & Solute carrier family 5 (sodium/myo-inositol cotransporter), member 3 \\
AASS & Aminoadipate-semialdehyde synthase \\
RIMKLA & Ribosomal modification protein rimK-like family member A \\
NCRNA00201 & Non-protein coding RNA 201 \\
ZMYM2 & Zinc finger, MYM-type 2 \\
NXNL2 & Nucleoredoxin-like 2 \\
HK2 & Hexokinase 2 \\
AHNAK & AHNAK nucleoprotein \\
ANKRD37 & Ankyrin repeat domain 37 \\
DEPP1 & DEPP1, autophagy regulator \\
PHF14 & PHD finger protein 14 \\
\hline
\end{tabular}

importance of FAO in their adaptation to hypoxia (17). It is plausible that FAO is a candidate therapeutically targeted pathway.

In conclusion, culture under mild hypoxia led to FAOdependent and -independent adaptation of HCT116 cells in vitro as well as tumor aggressiveness in vivo. The results provide insights into how hypoxia contributes to tumor progression, suggesting potential application of FAO to targeting hypoxic tumor cells.

\section{Conflicts of Interest}

The Authors declare that there are no potential conflicts of interest in regard to this study.

\section{Acknowledgements}

This work was supported in part by Japan Society for the Promotion of Science Grant-in-Aid for Scientific Research (C) 22501034 and 25430139, in part by Global COE Program for Metabolomics Systems Biology from the Ministry of Education, Culture, Sports, Science and Technology, and in part by School of Medicine, Keio University fund for the promotion of science.

The Authors thank Dr. Yotaro Izumi and Dr. Masato Nakamura for their insightful comments and suggestions.

\section{References}

1 Brown JM: Tumor hypoxia in cancer therapy. Methods Enzymol 435: 297-321, 2007.

2 Fluegen G, Avivar-Valderas A, Wang Y, Padgen MR, Williams JK, Nobre AR, Calvo V, Cheung JF, Bravo-Cordero JJ, Entenberg D, Castracane J, Verkhusha V, Keely PJ, Condeelis J and Aguirre-
Ghiso JA: Phenotypic heterogeneity of disseminated tumour cells is preset by primary tumour hypoxic microenvironments. Nat Cell Biol 19: 120-132, 2017.

3 Khatib AM, Fallavollita L, Wancewicz EV, Monia BP and Brodt P: Inhibition of hepatic endothelial E-selectin expression by cRAF antisense oligonucleotides blocks colorectal carcinoma liver metastasis. Cancer Res 62: 5393-5398, 2002.

4 Shen GM, Zhang FL, Liu XL and Zhang JW: Hypoxia-inducible factor 1-mediated regulation of PPP1R3C promotes glycogen accumulation in human MCF-7 cells under hypoxia. FEBS Lett 584: 4366-4372, 2010.

5 Passonneau JV and Lauderdale VR: A comparison of three methods of glycogen measurement in tissues. Anal Biochem 60: 405-412, 1974.

6 Karlsson H, Fryknäs M, Larsson R and Nygren P: Loss of cancer drug activity in colon cancer HCT-116 cells during spheroid formation in a new 3-D spheroid cell culture system. Exp Cell Res 318: 1577-1585, 2012.

7 Riffle S, Pandey RN, Albert M and Hegde RS: Linking hypoxia, DNA damage and proliferation in multicellular tumor spheroids. BMC Cancer 17: 338, 2017.

8 Riffle S and Hegde RS: Modeling tumor cell adaptations to hypoxia in multicellular tumor spheroids. J Exp Clin Cancer Res 36: 102, 2017.

9 Kalluri R and Weinberg RA: The basics of epithelialmesenchymal transition. J Clin Invest 119: 1420-1428, 2009.

10 Suemizu H, Monnai M, Ohnishi Y, Ito M, Tamaoki N and Nakamura M: Identification of a key molecular regulator of liver metastasis in human pancreatic carcinoma using a novel quantitative model of metastasis in $\mathrm{NOD} / \mathrm{SCID} / \gamma$ cnull $(\mathrm{NOG})$ mice. Int J Oncol 31: 741-751, 2007.

11 Lo JF, Yu CC, Chiou SH, Huang CY, Jan CI, Lin SC, Liu CJ, $\mathrm{Hu}$ WY and $\mathrm{Yu} \mathrm{YH}$ : The epithelial-mesenchymal transition mediator S100A4 maintains cancer-initiating cells in head and neck cancers. Cancer Res 71: 1912-1923, 2011. 
12 Fiermonte G, Paradies E, Todisco S, Marobbio CM and Palmieri F: A novel member of solute carrier family 25 (SLC25A42) is a transporter of coenzyme A and adenosine 3',5'-diphosphate in human mitochondria. J Biol Chem 284: 18152-18159, 2009.

13 P Pescador N, Villar D, Cifuentes D, Garcia-Rocha M, OrtizBarahona A, Vazquez S, Ordoñez A, Cuevas Y, Saez-Morales D, Garcia-Bermejo ML, Landazuri MO, Guinovart J and del Peso L: Hypoxia promotes glycogen accumulation through hypoxia inducible factor (HIF)-mediated induction of glycogen synthase 1. PLoS One 5: e9644, 2010.

14 Zhang Y, Xu D, Huang H, Chen S, Wang L, Zhu L, Jiang X, Ruan X, Luo X, Cao P, Liu W, Pan Y, Wang Z and Chen Y: Regulation of glucose homeostasis and lipid metabolism by PPP1R3G-mediated hepatic glycogenesis. Mol Endocrinol 28: 116-126, 2014.
15 Aqius L: Glucokinase and molecular aspects of liver glycogen metabolism. Biochem J 414: 1-18, 2008.

16 De Francesco EM, Maggiolini M, Tanowitz HB, Sotgia F and Lisanti MP: Targeting hypoxic cancer stem cells (CSCs) with doxycycline: Implications for optimizing anti-angiogenic therapy. Oncotarget 8: 56126-56142, 2017.

17 Iwamoto H, Abe M, Yang Y, Cui D, Seki T, Nakamura M, Hosaka K, Lim S, Wu J, He X, Sun X, Lu Y, Zhou Q, Shi W, Torimura T, Nie G, Li Q and Cao Y: Cancer lipid metabolism confers antiangiogenic drug resistance. Cell Metab 28: 104-117, 2018.

Received November 2, 2018

Revised November 21, 2018

Accepted November 22, 2018 